\title{
Physical Activity Classification Using Resilient Backpropagation (RPROP) with Multiple Outputs
}

\author{
Mustapha Maarouf and Blas J. Galván-González
}

Instituto de Sistemas Inteligentes y Aplicaciones Numéricas en Ingeniería (IUSIANI),

División de Computaciń Evolutiva y Aplicaciones (CEANI),

Universidad de Las Palmas de Gran Canaria, Islas Canarias, Spain

http://www. ceani.siani.es

\begin{abstract}
Considerable research has been conducted into the classification of Physical activity monitoring, an important field in computing research. Using artificial neural networks model, this paper explains novel architecture of neural network that can classify physical activity monitoring, recorded from 9 subjects. This work also presents a continuation of benchmarking on various defined tasks, with a high number of activities and personalization, trying to provide better solutions when it comes to face common classification problems. A brief review of the algorithm employed to train the neural network is presented in the first section. We also present and discuss some preliminary results which illustrate the performance and the usefulness of the proposed approach. The last sections are dedicated to present results of many architectures networks. In particular, the experimental section shows that multiple-output approaches represent a competitive choice for classification tasks both for biological purposes, industrial etc.
\end{abstract}

Keywords: Resilient Backpropagation, Classification, Physical Activity Monitoring, Activity Recognition.

\section{Introduction}

Recently, data sets from different fields of activity and recognition have become publicly. Monitoring objectives are to estimate physical activity intensity and recognize activities. The approach and challenges in this field are the different conditions, monitoring and the difficulty of common classification problems. This area of research suffers from many problems; there is a deficient of standard dataset, due to differing physical conditions of individuals, and also established benchmarking problems. However, only a few limited datasets are publicly available in this field of research [1]. Hence, there is a need for datasets specifically created for physical activity monitoring, and benchmarked on tasks defined in this field. However, only a few, limited datasets are publicly available in this research field. 
Physical activity has been identified as one of three key health behaviors impacting the major chronic diseases of aging that are increasingly responsible for a substantial proportion of global mortality, the goals of physical activity monitoring are to estimate the intensity and to recognize activities like sitting, walking, running or cycling, etc.

Physical activity has been identified as one of three key health behaviors impacting the major chronic diseases of aging that are increasingly responsible for a substantial proportion of global mortality.

\section{Problem Description and Review of Existing Approaches}

It's important to note that the detection of relevant actions in real life is necessary in many areas. the works of [1],[3],[4],[5] have presented several works, for the classification of physical activities, using various techniques of classification and recognition of activities.

However, usually tests and algorithms are performed in their own databases using particular specifications related to research groups. For this reason, its difficult to compare and conclude which methodology or technique is better. Therefore there is a need for databases that allow the comparison of different learning algorithms under the same conditions. This would allow replication of the procedures using different approaches, and should be flexible allowing different experimental setups.

In [9] illustrates the use of the dataset addressing the recognition of gestures and modes of locomotion using body-worn sensors for comparing different techniques by presenting a benchmarking study of four classification techniques (K-nearest neighbors, Nearest Centroid Classier, Linear and Quadratic Discriminant Analysis). In [1] have used five different classifiers selected from the Weka toolkit for creating the benchmark: Decision tree (C4.5), Boosted C4.5 decision tree, Bagging $\mathrm{C} 4.5$ decision tree, Nave Bayes and KNN. It has achieved good ( $90 \%$ and more) performance using the $\mathrm{KNN}$ and the boosted decision tree classifiers, but when comparing classification performance individually for the 9 subjects, a high variance can be observed: the individual performance varies on the all task between $74.02 \%$ and $100 \%$. Therefore, personalization approaches (subject dependent training) could significantly improve on the results of the benchmark, and are highly encouraged.

The intensity estimation and activity recognition tasks can be regarded as classification problems.

In our experiment, we will try to achieve better results considering classification with all task at the same time.

\section{Artificial Neural Nestworks Algorithms}

\subsection{Introduction}

The power and utility of artificial neural networks (ANNs) have been shown in several applications including speech synthesis, diagnostic problems, medicine, 
business and finance, control, robotics, signal processing, computer vision and many other problems that are included in the category of pattern recognition. In some application areas, neural models are promising to achieve human-like performance through artificial intelligence techniques more traditional.

Nowadays significant progress has been made in the field of neural networks, enough to attract a lot of attention and research funding. Research in the field is advancing on many fronts. New neural concepts are emerging and applications to complex problems developing. Clearly, today is a period of transition for neural network technology.

A neural network is a powerful data modeling tool that is able to capture and represent complex relationships between inputs and outputs. The motivation for the development of neural network technology stemmed from the desire to develop an artificial system that could perform "intelligent" tasks similar to those performed by the human brain. The models of ANNs are being applied successfully for solving a variety of complex problems in various fields of research.

The Backpropagation algorithm is a learning algorithm used to train networks with multiple layers by minimizing propagation error by gradient descent method that iteratively adjusts the parameters of the neural network (weights) to minimize the sum of errors The choice of the learning rate, which scales the derivative, has a significant effect on the time required to achieve the convergence. If set too small, too many steps are necessary to reach an acceptable solution, instead a possibly large learning rate will lead to oscillations, preventing the error falls below a certain value. To overcome these inherent disadvantages, many algorithms have been proposed to deal with the problem, doing some kind of adaptation of parameters during learning (e.g. global and local adaptive algorithms) [6].

\subsection{Reslient Backpropagation}

The resilient Backpropagation (RPROP) algorithm proposed by Riedmiller and Braun is one of the best performing learning methods for neural networks. RPROP is an effective new learning that performs a direct adaptation of the weight scale information based on the local gradient. A fundamental difference with adaptive techniques previously developed, the adaptation effort does not tarnish the gradient behavior whatsoever [6]. In [7] Present description of RPROP algorithm and implementation details. We propose a novel neural using resilient Backpropagation to train, with different architectures, is performed on classification problem. Our dataset for physical activity monitoring, recorded from 9 subjects, performing 18 different activities [1]-[2], are publicly available, and can be downloaded from [8].

In the algorithm 1 we describe the Rprop algorithm as proposed in [6]. The basic principal of RPROP is the direct adaptation of the weight update values $\Delta_{i j}$, in contrast to learning rate employed with gradient descent. With the gradient descent learning, the size of the derivative decreases exponentially, but the size of the weight step is only dependent on the sequence of signs and not on the magnitude of the derivative using RPROP. 




\subsection{Neural Network Implementation}

There is a wide variety of literature available in the field of artificial intelligence. Usually one of the main concerns is to identify network settings for our application. The important points to consider for this are:

- Identification of the input variables.

- Network type and transfer / activation function.

- Number of training patterns.

- Network structure (number of layers, the number of neurons, the number of neurons in each layer).

- Training.

- Validation of the network.

As we need to define a good set of training data to the neural network, we have been made data mining process that we can explain briefly in the following steps:

- Data Preparation: general cleaning (removing bad data measured, and complementing the missing data with the basic methods of similarity, and statistics, if necessary, the potential impact they can have missing data on the representativeness of the data analyzed).

- Selecting the data set: the target variables (those that we want to classify)

- Extract the significant periods of the output variables.

Once we have defined the training data, we have adopted various architectures to define how many hidden neurons and hidden layers should have our neural network. 


\section{Simulation and Results}

We have addressed the problem of classifying the 18 different physical activities (such as walking, cycling, playing soccer, etc.), performed by 9 subjects wearing 3 inertial measurement units and a heart rate monitor [8], without having to reduce the input variables, or discarding some activity. This has not been carried out in previous work due to the difficulties of the classification by increasing number of activities and the size of data to be used during the training and the evaluation of our method. The power of RPROP is assuring the convergence in a few time steps.

The best neural network architecture, having varied the number of hidden layers, is just with only three layers (the first layer for input, one hidden layer, and the last is for output neurons). Activation function which has been used to modify the weights is the hyperbolic tangent.

We performed two types of neural architectures using RPROP as a training method.

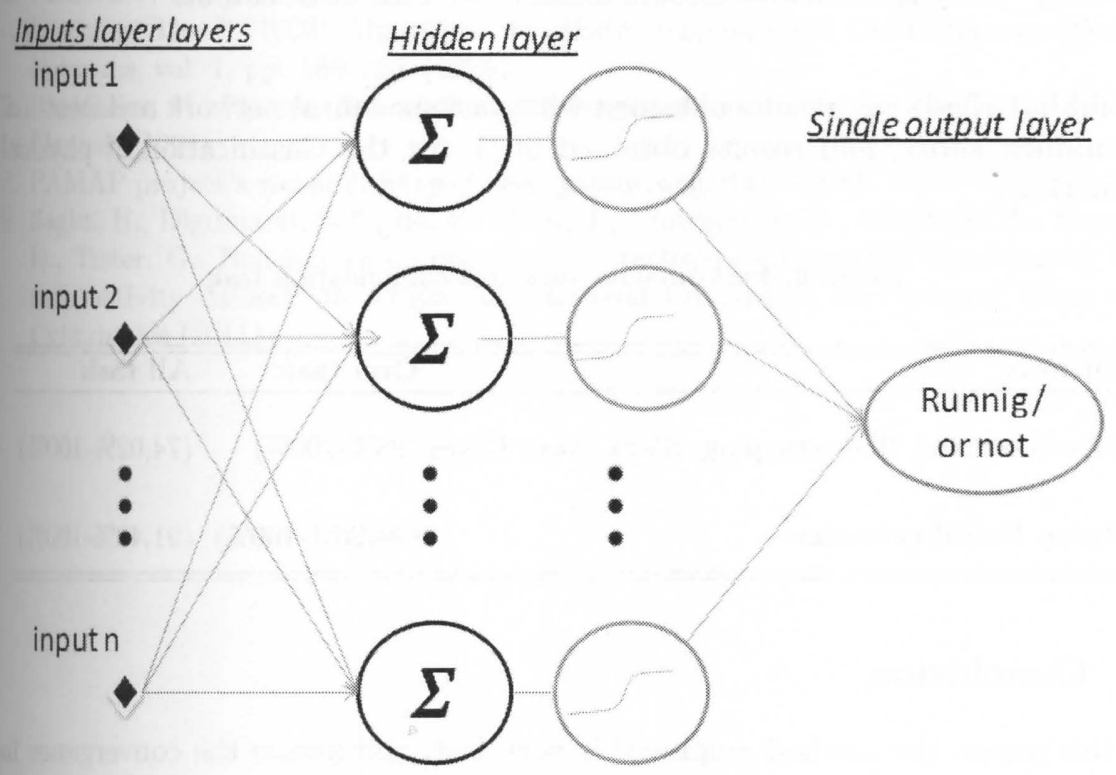

Fig. 1. Neural network architecture with single-outputs

The first is to classify only if it is a specific activity or not. In Fig.1, we can see an example of a neural network to classify if the individual is running or not. The same architecture has been used to the others activities.

And the second architecture aims to classify over all the activities which is of them are the objective (see Fig.2). 


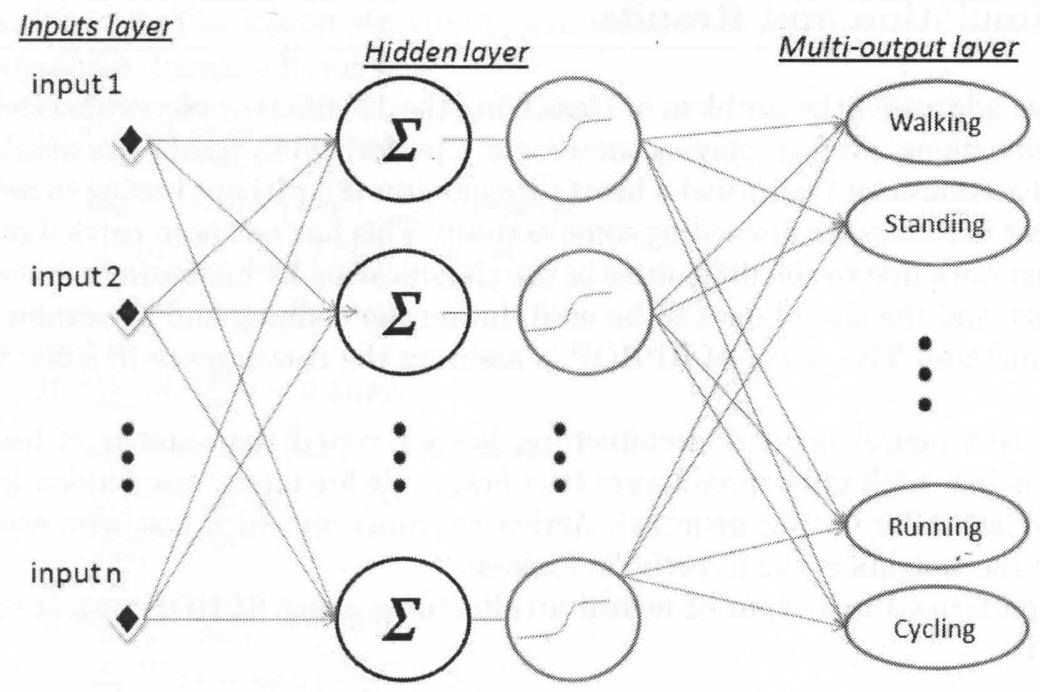

Fig. 2. Neural network architecture with multi-outputs

Table 1 shows the results obtained with various neural network architectures mentioned above, and results obtained in [1] for the classification of physical activities.

Table 1. Performance measures classification task

\begin{tabular}{lcc}
\hline \hline Methods & One task & All task \\
\hline [1]Decision tree, Bootstrapping, SVM, Nave Bayes & $(93 \%-100 \%)$ & $(74,02 \%-100 \%)$ \\
Rprop Neural networks & $(98,26 \%-100 \%)$ & $(91,48 \%-100 \%)$ \\
\hline \hline
\end{tabular}

\section{Conclusion}

In this paper, the method employed is very fast, and assure the convergence in the training step. The classification scheme was tested with experimental data collected in [1]-[2]. The classification was successfully realized and the performance varies between $98,26 \%$ and $100 \%$ to classify one activity and between $91,48 \%$ and $100 \%$ to classify all the activity, is an improvement of the results obtained in [1]. Nevertheless, more algorithms and techniques should be investigated in future work, combining different features.

Another point to be noted is to enlarge the dataset, and to look if there is a way to create a universal dataset for comparing and test with another results and techniques. 
Acknowledgements. The authors would like to thank the project PAMAP for the intention of offering a open database, to learn and share knowledge.

\section{References}

1. Reiss, A., Stricker, D.: Creating and Benchmarking a New Dataset for Physical Activity Monitoring. In: PETRA 2012, Crete Island, Greece, June 6-8 (2012)

2. Reiss, A., Stricker, D.: Towards Global Aerobic Activity Monitoring. In: 4th International Conference on Pervasive Technologies Related to Assistive Environments "PETRA" (2011)

3. Bao, L., Intille, S.: Activity recognition from user-annotated acceleration data. In: Proc. 2nd Int. Conf. Pervasive Comput., pp. 1-17 (2004)

4. Reiss, A., Weber, M., Stricker, D.: Exploring and Extending the Boundaries of Physical Activity Recognition. In: IEEE SMC Workshop on Robust Machine Learning Techniques for Human Activity Recognition, pp. 46-50 (2011)

5. Xue, Y., Jin, L.: A Naturalistic 3D Acceleration-Based Activity Dataset \& Benchmark Evaluations. In: International Conference on Systems, Man and Cybernetics (SMC), pp. 4081-4085 (2010)

6. Riedmiller, M., Braun, H.: A Direct Adaptive Method for Faster Backpropagation Learning: The RPROP Algorithm. In: IEEE International Conference on Neural Networks, vol. 1, pp. 586-591 (1993)

7. Riedmiller, M.: Rprop - Description and Implementation Details. Technical Report (January 1994)

8. PAMAP project's website, http://www.pamap.org/demo.html

9. Sagha, H., Digumarti, S.T., del R. Milln, J., Chavarriaga R., Calatroni A., Roggen D., Trster, G.: Benchmarking classification techniques using the Opportunity human activity dataset. In: IEEE International Conference on Systems, Man, and Cybernetics (2011) 